\title{
AN EXTENSION OF A RESULT OF CSISZAR
}

\author{
P. B. CERRITO \\ Department of Mathematics \\ University of South Florida \\ Tampa. Florida 33620 \\ (Received July 22, 1985)
}

ABSTRACT. We extend the results of Csiszar (z. Wahr. 5(1966) 279-295) to a topological semigroup $S$. Let $\mu$ be a measure defined on $S$. We consider the value of $\alpha=$ sup $1 \mathrm{~lm}$ sup $\mu^{n}\left(K x^{-1}\right)$. First. we show that the value of $\alpha$ is either compact

zero or one. If $\alpha=1$. we show that there exists a sequence of elements $\left(a_{n}\right.$ ) in $S$ such that $\mu^{n} * \delta_{a_{n}}$ converges vaguely to a probability measure where $\delta$ denotes point mass. In particular, we apply the results to inverse and matrix semigroups.

KEY WORDS AND PHRASES. TOPOlogical semigroup. Inf inite convolutions. PRIMARY CLASSIFICATION. $\quad$ GOF 17

1. INTRODUCTION.

Csiszar [1] proved the following result concerning a regular probability measure $\mu$ on a locally compact, second countable. Hausdorff group G: Either sup $\mu^{n}\left(K^{-1}\right) \rightarrow 0$ as $n \rightarrow \infty$ for all compact sets $K$. where $\mu^{n}$ denotes the $n$-fold convolution of $\mu$. or there exists a sequence of elements $\left\{a_{n}\right\}$ such that $\mu^{n} * \sigma_{a_{n}}$ converges vaguely to a probability measure where $\delta_{a_{n}}$ denotes point mass at $a_{n}$. We will extend this result to probability measures defined on certain types of locally compact. second countable. Hausdorff semigroups which satisfy condition (c): If $A$ and $B$ are compact then so are $A B^{-1}$ and $A^{-1} B$ where

$$
A B^{-1}=\{y: \text { there exists } z \in B \text { such that } y z \in A\} \text {. }
$$


We will also consider $\mu^{n}\left(\mathrm{Kx}^{-1}\right)$ when $\mu$ is defined on a semigroup $s$ $m \times m$ matrices. A matrix semigroup does not necessarily satisfy condition (c).

To each regular probability measure $\mu$ on a semigroup $S$ we associate the value $\alpha_{0}=\sup _{K} \lim _{n \rightarrow \infty} \sup _{x \in S} \mu^{n}\left(K^{-1}\right)$. We first show that $\alpha_{0}=0$ or $\alpha_{0}=1$. If compact

$a_{0}=0$ then $\mu^{n} * \delta_{a_{n}} \rightarrow 0$ vaguely for any sequence of elements $\left\{a_{n}\right.$ \} in $s$. If $a_{0}=1$ we find $\left\langle a_{n}\right\rangle$ such that $\mu^{n} * \delta_{a_{n}}$ converges to a probability measure.

2. PRELIMINARY RESULTS.

In order to show the main results. we need the following lemma. We omit its proof since it is quite similar to an argument of Csiszar [1].

LEMMA 1. Assume $S$ satisfies condition (c). Let $\mu_{1}$ be a probability measure such that sup $\mu_{1}\left(k x^{-1}\right) \leq \alpha$ for a compact set $K \subset s$. Then there exists a compact set $K_{2}$ (depending on $\mu_{1}$ ) such that for any other probability measure on $S$.

$$
\mu_{1} * \mu_{2}\left(k x^{-1}\right) \leq \alpha-\alpha 2\left(1-\mu_{2}\left(k_{2} x^{-1}\right)\right)
$$

Def ine $\alpha_{n}(k)=\sup _{x} \mu^{n}\left(K x^{-1}\right)$. Then if $k<n$.

$$
\begin{aligned}
\mu^{n}\left(K x^{-1}\right) & =\int \mu^{k}\left(K x^{-1} y^{-1}\right) \mu^{n-k}(d y) \\
& \leq \alpha_{k}(k) \int \mu^{n-k}(d y)=\alpha_{k}(k) .
\end{aligned}
$$

Therefore $\left\langle\alpha_{n}(k)\right\rangle$ is a nonincreasing sequence. Define $a(k)=\underset{n \rightarrow \infty}{\lim } \alpha_{n}(k)$ and $\alpha_{0}=\sup _{K} \alpha(K)$.

compact

THEOREM 1. If $s$ satisfies condition (c) then either $\alpha_{0}=0$ or $\alpha_{0}=1$.

PROOF. Suppose $0<\alpha_{0}<1$. Then there exists an $\alpha$ such that

$0<\alpha(1+\alpha) / 2<\alpha_{0}<\alpha<1$. For any compact set $k$ there exists a $k(k)$ such that sup $\mu^{k}\left(K x^{-1}\right)<a$. Applying Lemma 1 to $\mu_{1}=\mu^{k}$ and $\mu_{2}=\mu^{n-k}$ yields the fact that for some $K_{2}$.

$$
\mu^{n}\left(k x^{-1}\right) \leq \alpha-\alpha 2\left(1-\mu^{n-k}\left(k_{2} x^{-1}\right)\right)
$$

If $n$ is sufficiently large. $\mu^{n-k}\left(k_{2} x^{-1}\right)<\alpha$ for all $x$ since

$$
\sup \mu^{n-k}\left(k_{2} x^{-1}\right)<\alpha\left(k_{2}\right) \leq \alpha_{0}<\alpha .
$$

But then

$$
\mu^{n}\left(K x^{-1}\right) \leq \alpha-\alpha / 2(1-\alpha)=\alpha(1+\alpha) / 2 .
$$


Therefore $\alpha(K) \leq \alpha(1+\alpha) / 2$. Since $K$ is arbitrary we have a contradiction. We conclude that $\alpha_{0}=0$ or $\alpha_{0}=1$.

Before proceeding we present an example. Let $S=[0 . \infty)$ with the usual topology. Define multiplication by $r \cdot s=\max (r . s)$. Let $K=[0, n]$ be a compact subset of $s$. Then

$$
K x^{-1}=\left\{\begin{array}{lll}
0 & x>n \\
k & x \leq n
\end{array} \text { and } \mu^{n}\left(K x^{-1}\right)=\left\{\begin{array}{lll}
0 & x>n \\
\mu(k)^{n} & x \leq n
\end{array}\right.\right.
$$

Therefore if $\mu$ has compact support then $\alpha_{0}=1$. Otherwise. $\alpha_{0}=0$.

3. MATRIX SEMIGROUPS

Let $S$ be the set of all $\mathrm{m} \times \mathrm{m}$ matrices with probability measure $\mu$ defined on $S$ such that the support of $\mu$ generates a subsemigroup $s_{\mu}$ of $s$. We assume $s$ has the usual topology. Define $G=\langle X \in S: X$ is nonsingular $\rangle$. Then $G$ forms a subgroup of $S$. We want to consider the subgroup $G_{\mu}$ of $G$ generated by the set $s_{\mu} \cap G$. We consider the case where $G_{\mu}$ is locally compact. Then $G_{\mu}$ becomes a topological subgroup of $S$. If $\mu(G)=1$ then we need only apply Csiszar [1] to show that $\alpha_{0}=0$ or $\alpha_{0}=1$. Therefore we assume $0<\mu(G)<1$. Define a measure $\mu^{\cdot}$ on $G$ such that

$$
\mu^{\cdot}(B)=\mu(B \cap G) / \mu(G) \text { for } B \subset S .
$$

Then $\left(\mu^{\cdot}\right)^{2}(\mathrm{~B})=\int_{S} \mu^{\cdot}\left(\mathrm{Bx}^{-1}\right) \mu^{\cdot}(\mathrm{dx})$

$$
\begin{aligned}
& =\int_{G} \mu\left(B x^{-1} \cap G\right) / \mu(G) \mu \cdot(d x) \\
& =1 / \mu(G) \int_{G} \mu\left(B x^{-1} \cap G\right) / \mu(G) \mu(d x)
\end{aligned}
$$

Now $B x^{-1} \cap G=\langle y \in G: y x \in B\rangle=\langle y \in S: y x \in B \cap G\rangle$ if $x \in G$. Therefore.

$$
\left(\mu^{\cdot}\right)^{2}(\mathrm{~B})=1 / \mu(\mathrm{G})^{2} \int_{G} \mu\left((\mathrm{B} \cap \mathrm{G}) \mathrm{x}^{-1}\right) \mu(\mathrm{dx})
$$

If $x \in G$ then $(B \cap G) x^{-1}=0$. Therefore

$$
\begin{aligned}
\left(\mu^{\cdot}\right)^{2}(B)= & 1 / \mu(G)^{2} \int_{S} \mu\left((B \cap G) x^{-1}\right) \mu(d x) \\
& =\mu^{2}(B \cap G) / \mu(G)^{2} .
\end{aligned}
$$

By an induction argument.

$$
\left(\mu^{\cdot}\right)^{n}(B)=\mu^{n}(B \cap G) / \mu(G)^{n}
$$


Def ine the following notation:

$$
\begin{aligned}
a_{g} & =\sup _{K \in G} 11 \mathrm{~m} \sup _{x \in G}\left(\mu^{\cdot}\right)^{n}\left(K x^{-1}\right) \\
& =\sup _{K \in G} 11 \mathrm{~m} \sup _{x \in S}\left(\mu^{\cdot}\right)^{n}\left(K x^{-1}\right) \\
& =\sup _{K \in G} 1 \mathrm{im} \underset{x \in S}{\left[\sup ^{n} \mu^{n}\left(K x^{-1}\right)\right] / \mu(G)^{n} .}
\end{aligned}
$$

Since $\mu(G)<1 . \mu(G)^{n} \rightarrow 0$ as $n \rightarrow \infty$. By Csiszar's result [1] for groups. either $\alpha_{g}=0$ or $\alpha_{g}=1$. However.

$$
\lim \left[\sup \mu^{\mathrm{n}}\left(\mathrm{Kx}^{-1}\right)\right] \mu(G)^{\mathrm{n}}<\infty
$$

This is only possible if 1 im sup $\mu^{n}\left(\mathrm{Kx}^{-1}\right)=0$ for any $\mathrm{K} \subset \mathrm{G}$. Henceforth. we assume that $K$ is a compact set consisting of singular matrices. We will also exclude the zero matrix from our discussion since $0^{-1} O=S$ reduces the problem to a triviality and 1 t is obvious that $\alpha_{0}=1$. That is, we define

$$
\alpha_{0}=\sup _{K \subset S} 12 \mathrm{~m} \sup _{\substack{x \in S \\ x \neq 0}} \mu^{n}\left(\mathrm{Kx}^{-1}\right)
$$

We give an example. Suppose $s_{\mu}$ consists of matrices with nonnegative entries such that for any $x \in S_{\mu}$. every entry in $x$ is contained in the set $[0 . \infty)$ where $\delta>1 / \mathrm{m}$. Then

$$
\mu^{n+1}\left(K x^{-1}\right)=\int \cdots \int \mu\left(k\left(y_{n} \cdots y_{1} x\right)^{-1}\right) \mu\left(d y_{1}\right) \cdots \mu\left(d y_{n}\right)
$$

where $k\left(y_{n} \cdots y_{1} x\right)^{-1}=\left\langle z \in S: z y_{n} \cdots y_{1} x \in k\right\rangle$ and

$$
y_{n} \cdots y_{1} x=\left[\begin{array}{ccc}
w_{11} & \cdots & w_{1 m} \\
\vdots & & \\
w_{m 1} & \cdots & w_{m m}
\end{array}\right]
$$

where $w_{1 j}$ has minimal value $m^{n-1} \delta^{n}$ for all $i$ and $j$. Therefore for $z=\left(z_{i j}\right) \in k\left(y_{n} \cdots y_{1} x\right)^{-1} \cdot m^{n-1} \delta^{n} \sum z_{i j} \in k$ so that as $n \rightarrow \infty$. $\sum z_{i j} \rightarrow 0$ for all i. Hence for any compact set $K$.

$$
\lim _{n} \mu^{n}\left(K x^{-1}\right)=0
$$

and $\alpha_{0}=0$. By a similar argument. if every entry of $x \in s_{\mu}$ is contained in $[0.1 / m)$. then $\alpha_{0}=1$. 
In order to state a more general result. it is necessary to define some notation. Let $\Delta_{k}$ be the diagonal idempotent matrix of rank $k$. Let $y_{1} \cdot y_{2} \cdot \cdots \cdot y_{n} \in s_{\mu} \cdot$ Then

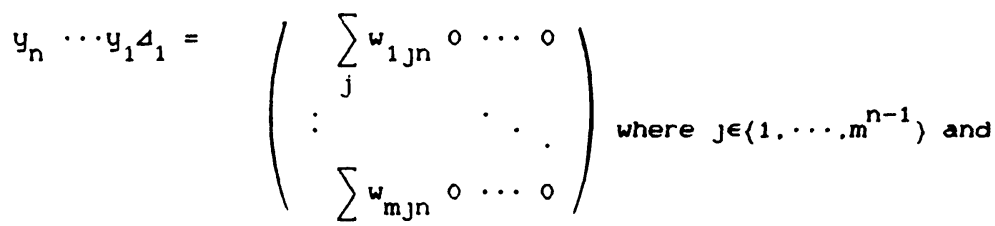

$w_{1 \text { jn }}$ represents the product of $n$ real numbers. We need to consider the distribution of $s_{n_{1}}=\sum_{j} w_{1 j n}$ where $j \in\left\langle 1,2, \cdots, m^{n-1}\right\}$. Let $F_{1 j n}$ be the distribution function of the random variable $w_{1 \mathrm{jn}} \cdot i=1.2, \cdots \cdot \mathrm{m}$ : $J=1.2, \cdots \cdot m^{n-1}: n=1.2 \ldots$. If we assume independence between the entries in the matrices then we may apply the Lindberg-Feller Theorem [2] to the double array $\left\langle w_{1 \mathrm{jn}}\right\rangle_{\mathrm{jn}}$ for every 1 .

THEOREM 2. Suppose the $\left\langle w_{1 \mathrm{jn}}\right\}_{\mathrm{jn}}$ def ined above satisfy the following conditions for each i:

1. $\sum_{j} \operatorname{var}\left(w_{i j n}\right)=1$ for every $n$.

2. $E\left(w_{1, j n}\right)=0$ for every J.n.

If $\sum \int y^{2} d F_{1 j n}(y) \rightarrow 0$ where the integral is taken over the set $\left|y^{2}\right|>\delta$ for each $0>0$ as $n \rightarrow \infty$ then $\alpha_{0}=1$.

PROOF: By the Lindberg-Feller Theorem. $S_{n i}$ converges in distribution to the standard normal for every 1. Therefore for $n$ and $N$ sufficiently large. $P\left(\left|S_{n_{1}}\right| \leq N\right)=1-\epsilon$ for all 1 where $\epsilon \rightarrow 0$ as $N \rightarrow \infty$. Therefore

$$
\begin{aligned}
\mu\langle x & \left.=\left(x_{i j}\right): x y_{1} \cdots y_{n} \Delta_{1} \in k_{k}=[-k, k]\right\} \\
& =\mu\left\langle x:\left|\sum_{1} x_{1 j} s_{n i}\right| \leq k \text { for all } j\right\rangle \\
& \geq \mu\left\langle x:\left|\sum_{1 j} x_{i j}\right| N \leq k \text { for all j }\right\}(1-\epsilon)^{m} \\
& \geq(1-\epsilon)^{\left.m_{\mu\left(k_{k}\right.}\right) .}
\end{aligned}
$$

Note that $k^{\cdot}$ depends only on the cholce of $N$ and $k$ and not on the choice of $n$.

Therefore as $k_{k} \uparrow S$ we may also let $N$ increase so it becomes clear that $\alpha_{0}=\sup \lim \mu^{n}\left(k \Delta_{1}^{-1}\right)=1$.

QED

It is clear that conditions (1) and (2) may be relaxed so that

$\sum_{j} \operatorname{Var}\left(w_{1 j n}\right)<M$ for some $M$ and $E\left(w_{1 j k}\right)<\infty$ for all $j . k$. 
We present an example. Suppose the support of the measure $\mu$.

$$
\begin{aligned}
& s_{\mu}=\left\{\left[\begin{array}{cccc}
x_{1} & x_{2} & x_{3} & x_{4} \\
0 & 0 & 0 & 0 \\
0 & 0 & 0 & 0 \\
0 & 0 & 0 & 0
\end{array}\right\}: x_{1} \in R=\right.\text { Then } \\
& \text { for any } x=\left[\begin{array}{cccc}
x_{1} & x_{2} & x_{3} & x_{4} \\
& 0 & &
\end{array}\right] \in s \mu \cdot x_{1}=\left[\begin{array}{cccc}
x_{1} & 0 & 0 & 0 \\
0 & & & \\
0 & 0 & \\
0 & &
\end{array}\right] \text {. }
\end{aligned}
$$

Therefore we need only be concerned with the probabllity distribution of the corner element. Suppose $x_{i j}$ is a random variable such that

$$
P\left(x_{i j}=1 / 2\right)=P\left(x_{i J}=-1 / 2\right)=1 / 2 \text { for all i.j. }
$$

Then $E\left(x_{1 j}\right)=0$ and $\operatorname{var}\left(x_{i j}\right)=1 / 4$. Also for any $n$. $E\left(x_{1 j} x_{2 j} \cdots x_{n j}\right)=0$ and $\operatorname{var}\left(x_{1 j} x_{2 j} \cdots x_{n j}\right)=1 / 4$. Define

$$
\begin{aligned}
w_{1 j 1} & =2 x_{1 j} \cdot j=1 \\
w_{1 j 2} & =2 x_{1 j} x_{2 j}, j=1.2 .3,4 \\
w_{1 j n} & =2 x_{1 j} x_{2 j} \cdots x_{n j} \cdot j=1.2, \cdots, 4^{n-1} . \text { Then } \sum \operatorname{var}\left(w_{1 j n}\right)=1 \text { and } \\
E\left(w_{1 j n}\right)=0 . ~ A l s o \int y^{2} d F_{1 j n}(y) & =0 \text { if } n \text { is sufficiently large. By the }
\end{aligned}
$$
above theorem. $\alpha_{0}=1$.

4. The case where $\alpha_{0}=1$.

If $\alpha_{0}=0$ then for all compact sets $K .1$ im sup $\mu^{n}\left(K x^{-1}\right)=0$ so that it is clear that for any sequence $\left\langle a_{n}\right\}, \mu^{n} * \delta_{\alpha_{n}}$ converges vaguely to the zero measure. Therefore we concentrate on the case where $\alpha_{0}=1$. Let $s$ be a locally compact. second countable. Hausdorff semigroup satisfying condition (c).

LEMMA 2. If $\alpha_{0}=1$ and $s$ is abelian then there exists a sequence $\left\langle x_{n}\right.$ ) such that for any $0 \leq \alpha<1$ there exists a compact set $k_{\alpha}$ such that $\mu^{n}\left(k_{\alpha} x_{n}^{-1}\right)>\alpha$ for all $\mathrm{n}$.

PROOF: For $\alpha=1 / 2$ there exists a $k_{2}$ such that $\sup _{x \in S} \mu^{n}\left(k_{2} x^{-1}\right)>1 / 2$ for all $n$. Therefore there exists a sequence $\left\langle x_{n}\right\}$ such that $\mu^{n}\left(k_{2} x_{n}^{-1}\right)>1 / 2$ for all $n$. Similarly. for each $\alpha>1 / 2$ there exists a $k_{\alpha}$ and a sequence $\left(x_{n \alpha}\right.$ ) such that $\mu^{n}\left(k_{\alpha} x_{n \alpha}^{-1}\right)>\alpha$. since $\alpha>1 / 2$. the sets $k_{2} x_{n}^{-1}$ and $k_{\alpha} x_{n \alpha}^{-1}$ cannot be disjoint so there must exist $w \in\left(k_{2} x_{n}^{-1}\right) \cap\left(k_{\alpha} x_{n \alpha}^{-1}\right)$. This implies that 
$x_{n \alpha} \in k_{\alpha} w^{-1} \subset k_{\alpha}\left(k_{2} x_{n}^{-1}\right)^{-1}$. Therefore $k_{\alpha} x_{n \alpha}^{-1} \subset k_{\alpha}\left\langle k_{\alpha}\left(k_{2} x_{n}^{-1}\right)\right]^{-1}$. suppose $y \in k_{\alpha} x_{n \alpha}^{-1}$. Then $y \in k_{\alpha}\left[k_{\alpha}\left(k_{2} x_{n}^{-1}\right)\right]^{-1}$ so there exists $z \in k_{\alpha}\left(k_{2} x_{n}^{-1}\right)^{-1}$ such that $y z \in K_{\alpha}$. Also $z \in K_{\alpha}\left(k_{2} x_{n}^{-1}\right)^{-1}$ implies there exists $z \cdot \in k_{2} x_{n}^{-1}$ such that $z z^{\cdot} \in k_{\alpha}$ and $z^{\cdot} x_{n} \in k_{2}$. Therefore $(y z)\left(z z^{\cdot}\right)\left(z^{\cdot} x_{n}\right) \in k_{\alpha}{ }^{2} k_{2}$. Since s is abelian. $y x_{n} \in\left(k_{\alpha}^{2}\right)^{-1} K_{\alpha}{ }^{2} k_{2}$ and $y \in\left(\left(k_{\alpha}^{2}\right)^{-1} k_{\alpha}{ }^{2} k_{2} x_{n}{ }^{-1}\right)$. By redefining $k_{\alpha}$ to be $\left(k_{\alpha}^{2}\right)^{-1} k_{\alpha}^{2} k_{2} \cdot \mu^{n}\left(k_{\alpha} x_{n}^{-1}\right)>\alpha$ for all $n$.

If $S$ is a group we can define $\nu_{n}=\delta_{x_{n-1}^{-1}} * \mu * \delta_{x_{n}}$ where the $x_{n}$ 's are defined in lemma 2. Then we can apply Csiszar [1] to $y_{k}^{n}=\nu_{k+1} * \nu_{k+2} * \cdots * \nu_{n}=\delta_{x_{k}^{-1}} * \mu^{n-k} * \delta_{x_{n}}$. Unfortunately $\delta_{x}-1$ has no meaning in a semigroup and the $\nu_{n}$ 's must be defined in some other way.

Suppose $S$ is embeddable in an abelian group $G$. Then by Lemma 2 there exists a sequence $\left\langle x_{n}\right.$ ) such that for any $\alpha$ there exists a $k_{\alpha}$ such that $\mu^{n}\left(k_{\alpha} x_{n}^{-1}\right)>\alpha$. We may assume that $\mu$ is a measure defined in $G$ with support contained in $S$. Then $\nu_{n}=\delta x_{n}^{-1} * \mu * \delta_{x_{n}}$ is well defined in $G$ if we let $x_{0}$ be the 1 dentity element of $G$. If we write $\left(\mathrm{Kx}^{-1}\right)_{S}$ and $\left(\mathrm{Kx}^{-1}\right)_{G}$ for the respective sets defined in $S$ and $G$ then $\left(\mathrm{Kx}^{-1}\right)_{S} \subset\left(\mathrm{Kx}^{-1}\right)_{G}$. However since the support of $\mu$ is contained in $S$. $\mu^{n}\left(\left(K x^{-1}\right)_{S}\right)=\mu^{n}\left(\left(K x^{-1}\right)_{G}\right)$. Therefore $\alpha_{0}=1$ with respect to $G$. Let $y_{k}^{n}=\nu_{k+1} * \cdots * \nu_{n}$. Then $y_{0}^{n}\left(k_{\alpha}\right)=\mu^{n}\left(k_{\alpha} x_{n}^{-1}\right)>\alpha$ for any $\alpha$. Also. by lemma 1 . $y_{k}^{n}\left(k_{\alpha}^{-1} k_{\alpha}\right) \geq y_{0}^{n}\left(k_{\alpha}\right)+y_{0}^{k}\left(k_{\alpha}\right)-1 \geq 2 \alpha-1$. Therefore 1 t is clear that any $11 \mathrm{~m} 1 \mathrm{t}$ point of $y_{k}^{n}$ must be a probability measure and Csiszar [1] can be applied to this sequence. It is also clear that any limit point of $y_{k}^{n}$ must have support contained in $S$ and may therefore be considered a measure on $S$.

Next consider the case where $S$ is an abelian inverse semigroup. $S$ is a semigroup of this type provided for any $x \in S$ there exists a unique $x^{\cdot} \in S$ such that $x x^{\prime} x=x$ and $x^{\prime} x x^{*}=x^{\cdot}$. A natural ordering can be defined on the idempotent elements of $S: e \leq f$ provided ef $=f e=e$. If $S$ contains a minimal idempotent e then we can define $\nu_{n}=\delta_{x_{n-1}} * \mu * \delta_{x_{n}}$ with $x_{0}=e$. Then $y_{0}^{n}\left(k_{\alpha} \cup k_{\alpha} e\right)=\nu_{1} * \cdots \nu_{n}\left(k_{\alpha} \cup k_{\alpha} e\right)$

$$
\begin{aligned}
& =\mu^{n}\left(\left(k_{\alpha} \cup k_{\alpha} e\right)\left(x_{n} e\right)^{-1}\right) \\
& \geq \mu^{n}\left(k_{\alpha} x_{n}^{-1}\right)>\alpha \text { for all } n .
\end{aligned}
$$

Therefore all limit points of $y_{k}^{n}$ are probabllity measures. 
If $s$ contains a finite number of idempotents. say $e_{1} \cdot e_{2} \cdot \cdots \cdot e_{n}$ then the product $e_{1} e_{2} \cdots e_{n}$ is minimal in $s$. Therefore csiszar [1] can be applied to any abelian inverse semigroup with a finite number of idempotents.

Suppose instead that $S$ is an inverse semigroup such that the set of idempotents can be ordered in the following manner: $f_{0}>f_{1}>f_{2}>\ldots$. That is. suppose $S$ is an w-semigroup. Let $x_{0}=f_{0}$ and consider the sequence $\left(x_{n}\right)$ defined in lemma 4 . Given any $x_{n}$ either

a. the 1dempotent $x_{j} x_{j}=e_{j}>x_{n} x_{n} \cdot=e_{n}$ for all $j>n$ or

b. there exists some $J>n$ such that $e_{j}<e_{n}$.

If there exists some $n$ for which (a) is true then $S$ has a minimal idempotent. If not. there exists a subsequence $x_{0} \cdot x_{i_{1}} \cdot x_{1_{2}} \cdots$ such that $e_{1}>e_{i_{n}}$ if $j>n$. Define $\nu_{n}=\delta_{x_{1 n-1}} * \mu^{1 n^{-1} n-1} * \nu_{x_{i}} e_{i_{n}}$.

THEOREM 3. If $y_{k}^{n}=\nu_{k+1} * \cdots * \nu_{n}$ is a sequence of probability measures on $s$ satisfying the hypotheses of Csiszar [1] then there exists a sequence $\left(w_{n}\right)$ in $s$ such that for each $k, y_{k}^{n} * \delta_{w_{n}}$ converges vaguely to a probabl11ty measure as $n \rightarrow \infty$.

PROOF. By Csiszar [1] there exists a sequence of integers $n_{1}<n_{2}<\cdots<n_{j}<\cdots$ such that

$$
\underset{j}{\lim y_{k}^{n}}=\lambda_{k} \text { and } \underset{j}{\lim \lambda_{n}}=\lambda_{\infty}
$$

where the $11 \mathrm{mits}$ are defined with respect to the vague topology and $\lambda_{k}$ is a probability measure for all $k \leq \infty$. Also $\lambda_{\infty}$ is 1 dempotent and $\lambda_{k} * \lambda_{\infty}=\lambda_{k}$ for al1 K.

The support of any idempotent probability measure is completely simple. Let $H$ denote the support of $\lambda_{\infty}$. Since $S$ is abelian. $H$ is a group. Furthermore. $\lambda_{\infty}$ is a Haar measure on $H$ and $H$ is a compact group.

The remainder of the proof. dealing with the cholce of a suztable sequence $\left\{w_{n}\right\}$. is quite similar to the argument in Csiszar [4] and will be omitted.

QED

We define $a_{n}=x_{n} w_{n}$ where $x_{n}$ is defined in lemma 2 and $w_{n}$ is defined above. If $S$ is embeddable or an inverse semigroup with a minimal idempotent then $\underset{n}{\operatorname{im}} y_{0}^{n} * \delta_{w_{n}}=1$ im $\mu^{n} * \sigma_{a_{n}}=\lambda_{0}$ which is a probability measure. In the other two cases. the same argument can be applied to an infinite subsequence. 


\section{REFERENCES}

1. CSISZAR. I. On Infinite Products of Random Elements and Infinite Convolutions of Probability Distributions on Locally Compact Groups. $z$. Wahr. verw. Geb. 5. 1966. 279-295.

2. CHUNG. K. L. A Course in Probability Theory. Academic Press. New York. 1970.

3. CLIFFORD. A. H. and PRESTON. G. B. The Algebraic Theory of Semigroups. Vol. I. Mathematical Surveys 7. Amer. Math. Soc.. Providience, R.I.. 1961.

4. CENTER. B. and MUKHERJEA. A. More on Limit Theorems for I terates of Probability Measures on Semigroups and Groups. Z. Wahr. verw. Geb. 46. 1979. 259-275.

5. MUKHERJEA. A. Limit Theorems for Convolution Iterates of a Probabilitity Measure on Completely simple or Compact Semigroups. Trans. Amer. Math. Soc.. 225. 1977. 355-370.

6. YEAGER. D. Imbedding compact Semigroups in compact Inverse semigroups. Semigroup Forum 10, 1975. 76-83. 


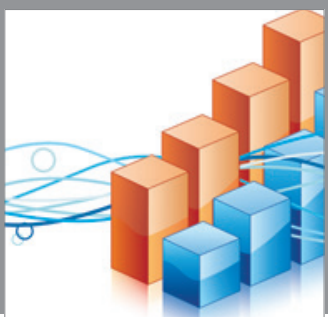

Advances in

Operations Research

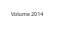

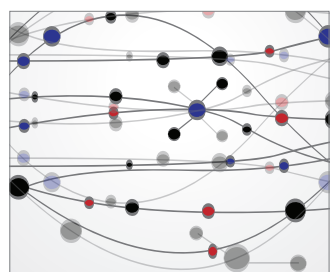

\section{The Scientific} World Journal
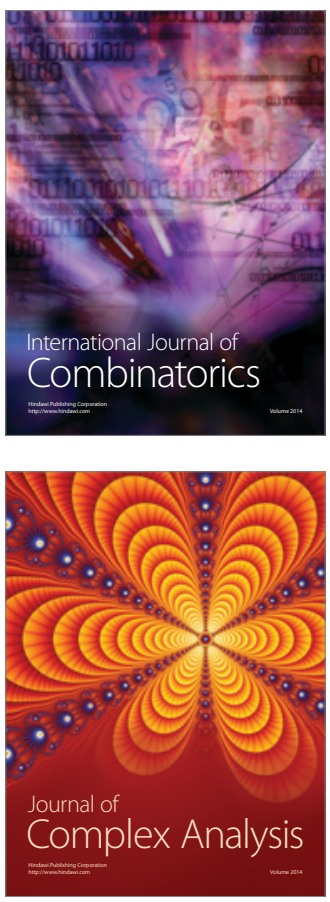

International Journal of

Mathematics and

Mathematical

Sciences
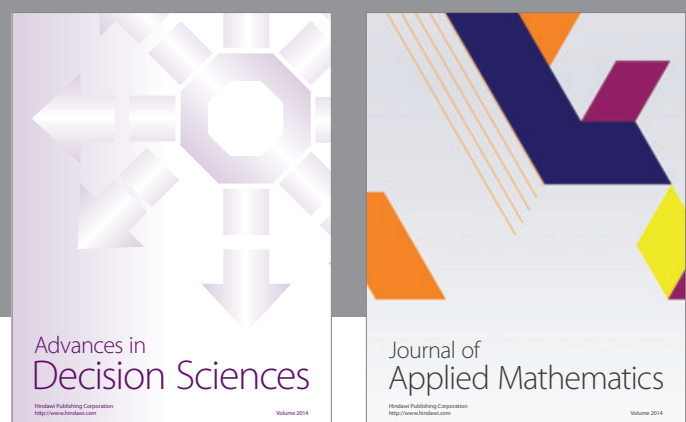

Journal of

Applied Mathematics
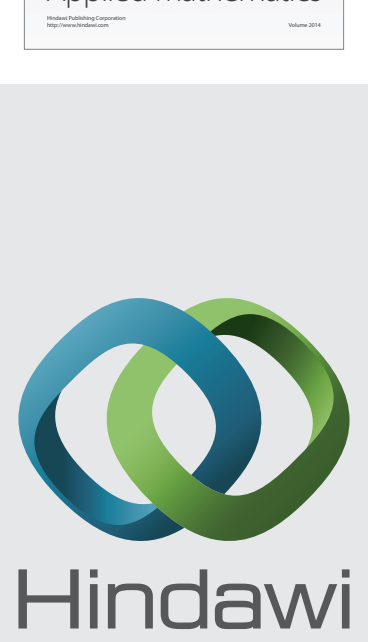

Submit your manuscripts at http://www.hindawi.com
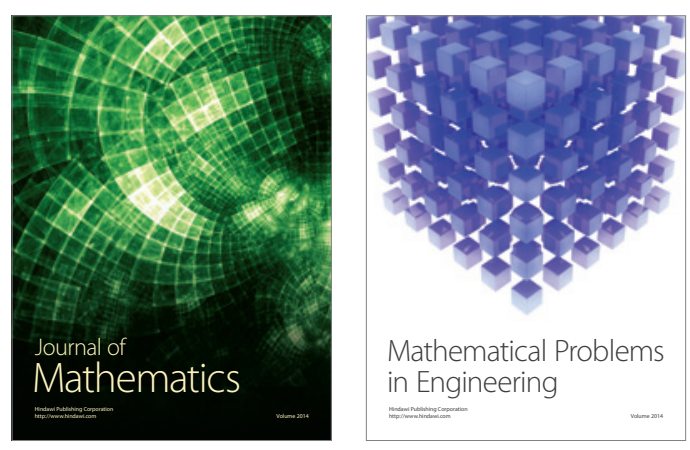

Mathematical Problems in Engineering
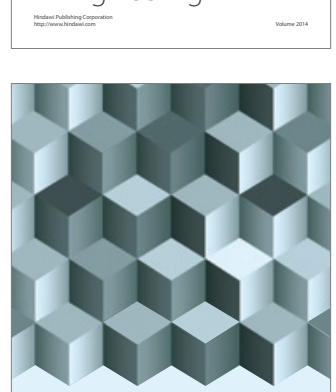

Journal of

Function Spaces
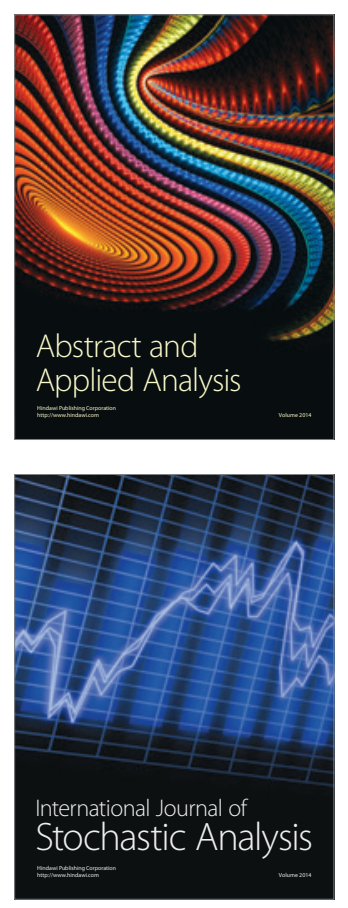

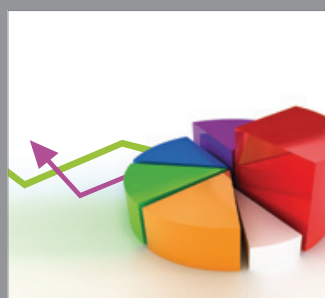

ournal of

Probability and Statistics

Promensencen
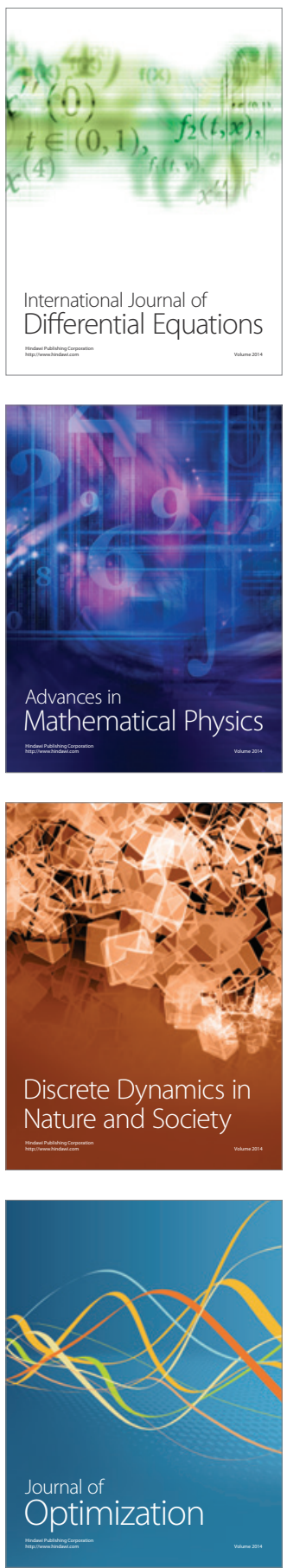